\title{
Inclusive fitness theory and eusociality
}

\author{
ARISING From M. A. Nowak, C. E. Tarnita \& E. O. Wilson Nature 466, 1057-1062 (2010)
}

Nowak et al. ${ }^{1}$ argue that inclusive fitness theory has been of little value in explaining the natural world, and that it has led to negligible progress in explaining the evolution of eusociality. However, we believe that their arguments are based upon a misunderstanding of evolutionary theory and a misrepresentation of the empirical literature. We will focus our comments on three general issues.

First, Nowak et al. ${ }^{1}$ are incorrect to suggest a sharp distinction between inclusive fitness theory and "standard natural selection theory". Natural selection explains the appearance of design in the living world, and inclusive fitness theory explains what this design is for. Specifically, natural selection leads organisms to become adapted as if to maximize their inclusive fitness ${ }^{2-4}$. Inclusive fitness theory is based upon population genetics, and is used to make falsifiable predictions about how natural selection shapes phenotypes, and so it is not surprising that it generates identical predictions to those obtained using other methods $\mathrm{s}^{2,5-7}$.

Second, Nowak et al. ${ }^{1}$ are incorrect to state that inclusive fitness requires a number of "stringent assumptions" such as pairwise interactions, weak selection, linearity, additivity and special population structures. Hamilton's original formulations did not make all these assumptions, and generalizations have shown that none of them is required $^{3,5,6,8}$. Inclusive fitness is as general as the genetical theory of natural selection itself. It simply partitions natural selection into its direct and indirect components.

Nowak et al. ${ }^{1}$ appear to have confused the completely general theory of inclusive fitness with models of specific cases. Yes, researchers often make limiting assumptions for reasons of analytical tractability when considering specific scenarios ${ }^{5,7}$, as with any modelling approach. For example, Nowak et al. ${ }^{1}$ assume a specific form of genetic control, where dispersal and helping are determined by the same single locus, that mating is monogamous, and so on. However, the inclusive fitness approach has facilitated, not hindered, empirical testing of evolutionary theory ${ }^{9-11}$. Indeed, an advantage of inclusive fitness theory is that it readily generates testable predictions in situations where the precise genetic architecture of a phenotypic trait is unknown.

Third, we dispute the claim of Nowak et al. ${ }^{1}$ that inclusive fitness theory "does not provide any additional biological insight", delivering only "hypothetical explanations", leading only to routine measurements and "correlative studies", and that the theory has "evolved into an abstract enterprise largely on its own", with a failure to consider multiple competing hypotheses. We cannot explain these claims, which seem to overlook the extensive empirical literature that has accumulated over the past 40 years in the fields of behavioural and evolutionary ecology ${ }^{9-11}$ (Table 1). Of course, studies must consider the direct consequences of behaviours, as well as consequences for relatives, but no one claims otherwise, and this does not change the fact that relatedness (and lots of other variables) has been shown to be important in all of the above areas.

We do not have space to detail all the advances that have been made in the areas described in Table 1. However, a challenge to the claims of Nowak et al. ${ }^{1}$ is demonstrated with a single example, that of sex allocation (the ratio of investment into males versus females). We choose sex allocation because: (1) Nowak et al. ${ }^{1}$ argue that inclusive fitness theory has provided only "hypothetical explanations" in this field; (2) it is an easily quantified social trait, which inclusive fitness theory predicts can be influenced by interactions between relatives; and (3) the study of sex allocation has been central to evolutionary work on the eusocial insects. In contrast to the claims of Nowak et al. ${ }^{1}$, recent reviews of sex allocation show that the theory explains why sex allocation varies with female density, inbreeding rate, dispersal rate, brood size, order of oviposition, sib-mating, asymmetrical larval competition, mortality rate, the presence of helpers, resource availability and nest density in organisms such as protozoan parasites, nematodes, insects, spiders, mites, reptiles, birds, mammals and plants ${ }^{5,12,13}$.

The quantitative success of this research is demonstrated by the percentage of the variance explained in the data. Inclusive fitness theory has explained up to $96 \%$ of the sex ratio variance in acrossspecies studies and $66 \%$ in within-species studies ${ }^{13}$. The average for all evolutionary and ecological studies is $5.4 \%$. As well as explaining adaptive variation in behaviour, inclusive fitness theory has even elucidated when and why individuals make mistakes (maladaptation), in response to factors such as mechanistic constraints ${ }^{13}$. It is not clear how Nowak et al. ${ }^{1}$ can characterize such quantifiable success as "meagre". Their conclusions are based upon a discussion in the Supplementary Information of just three papers (by authors who disagree with the interpretations of Nowak et al. ${ }^{1}$ ), out of an empirical literature of thousands of research articles. This would seem to indicate a failure to engage seriously with the body of work that they recommend we abandon.

The same points can be made with regard to the evolution of the eusocial insects, which Nowak et al. ${ }^{1}$ suggest cannot be explained by inclusive fitness theory. It was already known that haplodiploidy itself may have only a relatively minor bearing on the origin of eusociality, and so Nowak et al. ${ }^{1}$ have added nothing new here. Inclusive fitness theory has explained why eusociality has evolved only in monogamous lineages, and why it is correlated with certain ecological conditions, such as extended parental care and defence of a shared resource ${ }^{14,15}$. Furthermore, inclusive fitness theory has made very successful predictions about behaviour in eusocial insects, explaining a wide range of phenomena (Table 2).

Ultimately, any body of biological theory must be judged on its ability to make novel predictions and explain biological phenomena; we believe that Nowak et al. ${ }^{1}$ do neither. The only prediction made by their model (that offspring are favoured to help their monogamously

Table 1 Inclusive fitness theory has been important in understanding a range of behavioural phenomena

\begin{tabular}{llll}
\hline Research area & Correlational? & Experimental? & Theory-data interplay \\
\hline Sex allocation & Yes & Yes & Yes \\
Policing & Yes & Yes & Yes \\
Conflict resolution & Yes & Yes & Yes \\
Cooperation & Yes & Yes & Yes \\
Altruism & Yes & Yes & Yes \\
Spite & Yes & Yes & Yes \\
Kin discrimination & Yes & Yes & Yes \\
Parasite virulence & Yes & Yes & Yes \\
Parent-offspring conflict & Yes & Yes & Yes \\
Sibling conflict & Yes & Yes & Yes \\
Selfish genetic elements & Yes & Yes & Yes \\
Cannibalism & Yes & Yes & Yes \\
Dispersal & Yes & Yes & Yes \\
Alarm calls & Yes & Yes & Yes \\
Eusociality & Yes & Yes & Yes \\
Genomic imprinting & Yes & Yes & Yes \\
\hline
\end{tabular}

Data are taken from refs 9-11. Correlational studies test predictions using natural variation in key variables, whereas experimental studies involve their experimental manipulation. Interplay between theory and data means that theory has informed empirical study, and vice versa. Inclusive fitness is not the only way to model evolution, but it has already proven to be an immensely productive and useful approach for studying eusociality and other social behaviours. 
Table 2 | Areas in which inclusive fitness theory has made successful predictions about behaviour in eusocial insects

\begin{tabular}{|c|c|c|c|c|}
\hline Trait examined & Explanatory variables & $\begin{array}{l}\text { Correlational } \\
\text { studies? }\end{array}$ & $\begin{array}{l}\text { Experimental } \\
\text { studies? }\end{array}$ & $\begin{array}{l}\text { Interplay between } \\
\text { theory and data? }\end{array}$ \\
\hline Altruistic helping & Haplodiploidy versus diploidy & Yes & No & Yes \\
\hline Worker egg laying & Worker policing & Yes & Yes & Yes \\
\hline Policing & Relatedness & Yes & Yes & Yes \\
\hline Level of cooperation & Costs, benefits and relatedness & Yes & Yes & Yes \\
\hline Intensity of work & Need for work and probability of becoming queen & Yes & Yes & Yes \\
\hline Sex allocation & $\begin{array}{l}\text { Relatedness asymmetries due to variation in queen } \\
\text { survival, queen number and mating frequency }\end{array}$ & Yes & Yes & Yes \\
\hline Sex allocation & Resource availability & Yes & Yes & Yes \\
\hline Sex allocation & Competition for mates between related males & Yes & Yes & Yes \\
\hline Number of individuals trying to become reproductive & Presence of old queens & Yes & Yes & Yes \\
\hline Workers killing queens & Presence of workers, reproductives or other queens & Yes & No & No \\
\hline Exclusion of non-kin & Colony membership & Yes & Yes & Yes \\
\hline
\end{tabular}

Data are taken from refs $12-16$.

mated mother if this provides a sufficient benefit) merely confirms, in a less general way, Hamilton's original point: if the fitness benefits are great enough, then altruism is favoured between relatives.

Patrick Abbot ${ }^{1}$, Jun Abe ${ }^{2}$, John Alcock ${ }^{3}$, Samuel Alizon ${ }^{4}$, Joao A. C. Alpedrinha ${ }^{5}$, Malte Andersson ${ }^{6}$, Jean-Baptiste Andre ${ }^{7}$, Minus van Baalen ${ }^{7}$, Francois Balloux ${ }^{8}$, Sigal Balshine ${ }^{9}$, Nick Barton ${ }^{10}$, Leo W. Beukeboom ${ }^{11}$, Jay M. Biernaskie ${ }^{5}$, Trine Bilde ${ }^{12}$, Gerald Borgia $^{13}$, Michael Breed ${ }^{14}$, Sam Brown ${ }^{5}$, Redouan Bshary ${ }^{15}$, Angus Buckling ${ }^{5}$, Nancy T. Burley ${ }^{16}$, Max N. Burton-Chellew ${ }^{5}$, Michael A. Cant ${ }^{17}$, Michel Chapuisat ${ }^{18}$, Eric L. Charnov ${ }^{19}$, Tim Clutton-Brock ${ }^{20}$, Andrew Cockburn ${ }^{21}$, Blaine J. Cole ${ }^{22}$, Nick Colegrave ${ }^{23}$, Leda Cosmides ${ }^{24}$, Iain D. Couzin ${ }^{25}$, Jerry A. Coyne ${ }^{26}$, Scott Creel ${ }^{27}$, Bernard Crespi ${ }^{28}$, Robert L. Curry ${ }^{29}$, Sasha R. X. Dall ${ }^{17}$, Troy Day ${ }^{30}$, Janis L. Dickinson ${ }^{31}$, Lee Alan Dugatkin ${ }^{32}$,

Claire El Mouden ${ }^{5}$, Stephen T. Emlen ${ }^{33}$, Jay Evans ${ }^{34}$, Regis Ferriere ${ }^{35}$, Jeremy Field ${ }^{36}$, Susanne Foitzik ${ }^{37}$, Kevin Foster ${ }^{5}$, William A. Foster ${ }^{20}$, Charles W. Fox ${ }^{38}$, Juergen Gadau ${ }^{39}$, Sylvain Gandon ${ }^{40}$, Andy Gardner ${ }^{5}$, Michael G. Gardner ${ }^{41}$, Thomas Getty ${ }^{42}$, Michael A. D. Goodisman ${ }^{43}$, Alan Grafen ${ }^{5}$, Rick Grosberg ${ }^{44}$, Christina M. Grozinger ${ }^{45}$, Pierre-Henri Gouyon ${ }^{46}$, Darryl Gwynne ${ }^{47}$, Paul H. Harvey ${ }^{5}$, Ben J. Hatchwell ${ }^{48}$, Jürgen Heinze ${ }^{49}$, Heikki Helantera ${ }^{50}$, Ken R. Helms ${ }^{51}$, Kim Hill ${ }^{52}$, Natalie Jiricny ${ }^{5}$, Rufus A. Johnstone ${ }^{20}$, Alex Kacelnik ${ }^{5}$, E. Toby Kiers ${ }^{53}$, Hanna Kokko ${ }^{21}$, Jan Komdeur ${ }^{54}$, Judith Korb ${ }^{55}$, Daniel Kronauer ${ }^{56}$, Rolf Kümmerli ${ }^{57}$, Laurent Lehmann ${ }^{15}$, Timothy A. Linksvayer ${ }^{58}$, Sébastien Lion ${ }^{59}$, Bruce Lyon ${ }^{60}$, James A. R. Marshall ${ }^{61}$, Richard McElreath ${ }^{62}$, Yannis Michalakis ${ }^{4}$, Richard E. Michod ${ }^{63}$, Douglas Mock ${ }^{64}$, Thibaud Monnin ${ }^{7}$, Robert Montgomerie ${ }^{65}$, Allen J. Moore ${ }^{17}$, Ulrich G. Mueller ${ }^{66}$, Ronald Noë ${ }^{67}$, Samir Okasha ${ }^{68}$, Pekka Pamilo ${ }^{69}$, Geoff A. Parker ${ }^{70}$, Jes S. Pedersen ${ }^{58}$, Ido Pen ${ }^{71}$, David Pfennig ${ }^{72}$, David C. Queller ${ }^{73}$, Daniel J. Rankin ${ }^{74}$, Sarah E. Reece ${ }^{23}$, Hudson K. Reeve ${ }^{33}$, Max Reuter ${ }^{75}$, Gilbert Roberts ${ }^{76}$, Simon K. A. Robson ${ }^{77}$, Denis Roze ${ }^{78}$, Francois Rousset ${ }^{79}$, Olav Rueppell ${ }^{80}$, Joel L. Sachs ${ }^{81}$, Lorenzo Santorelli ${ }^{5}$, Paul Schmid-Hempel ${ }^{82}$, Michael P. Schwarz ${ }^{41}$, Tom Scott-Phillips ${ }^{83}$,

Janet Shellmann-Sherman ${ }^{33}$, Paul W. Sherman ${ }^{33}$, David M. Shuker ${ }^{84}$, Jeff Smith ${ }^{73}$, Joseph C. Spagna ${ }^{85}$, Beverly Strassmann ${ }^{86}$,

Andrew V. Suarez ${ }^{87}$, Liselotte Sundström ${ }^{50}$, Michael Taborsky ${ }^{88}$, Peter Taylor $^{30}$, Graham Thompson ${ }^{89}$, John Tooby ${ }^{90}$, Neil D. Tsutsui ${ }^{91}$, Kazuki Tsuji ${ }^{92}$, Stefano Turillazzi ${ }^{93}$, Francisco Úbeda ${ }^{94}$,

Edward L. Vargo ${ }^{95}$, Bernard Voelk| ${ }^{96}$, Tom Wenseleers ${ }^{97}$,

Stuart A. West ${ }^{5}$, Mary Jane West-Eberhard ${ }^{98}$, David F. Westneat ${ }^{99}$, Diane C. Wiernasz 22 , Geoff Wild ${ }^{100}$, Richard Wrangham ${ }^{101}$, Andrew J. Young ${ }^{17}$, David W. Zeh ${ }^{102}$, Jeanne A. Zeh ${ }^{102}$ \& Andrew Zink ${ }^{103}$

${ }^{1}$ Vanderbilt University, Nashville, Tennessee 37235, USA.
${ }^{2}$ Laboratory of Applied Entomology, Faculty of Agriculture, Shizuoka University, Sizuoka 422-8529, Japan.

${ }^{3}$ School of Life Sciences, PO Box 874501, Arizona State University, Tempe, Arizona 85287-4501, USA

${ }^{4}$ UMR CNRS-IRD 2724, Genetics and Evolution of Infectious Diseases, IRD, 911 Avenue Agropolis, B.P. 64501, 34394 Montpellier Cedex 5, France.

${ }^{5}$ Department of Zoology, University of Oxford, South Parks Road, Oxford, OX1 3PS, UK.

e-mail: Stuart.West@zoo.ox.ac.uk

${ }^{6}$ Department of Zoology, University of Gothenburg, SE 40530

Gothenburg, Sweden.

${ }^{7}$ CNRS, Université Pierre et Marie Curie, Ecole Normale Supérieure, UMR 7625, Ecologie and Evolution, 75005 Paris, France.

${ }^{8}$ MRC Centre for Outbreak Analysis and Modelling, Department of Infectious Disease Epidemiology, Faculty of Medicine, Imperial College, St Mary's Campus, Norfolk Place, London W2 1PG, UK.

${ }^{9}$ Department of Psychology, Neuroscience and Behaviour, McMaster University, 1280 Main St West, Hamilton, Ontario L8S 4K1, Canada.

${ }^{10}$ IST Austria, Am Campus 1, Klosterneuburg 3400, Austria.

${ }^{11}$ Evolutionary Genetics, Centre for Ecological and Evolutionary Studies, University of Groningen, PO Box 14, NL-9750 AA Haren, The Netherlands.

${ }^{12}$ Aarhus University, Department of Biological Sciences, Ny Munkegade 1540, 8000 Aarhus C, Denmark.

${ }^{13}$ Department of Biology, University of Maryland, College Park, Maryland 20742-4415, USA.

${ }^{14}$ Ecology and Evolutionary Biology, University of Colorado, Boulder, Colorado 80309-0334, USA.

${ }^{15}$ Faculté des sciences, Rue Emile-Argand 11, Case postale 158, 2000

Neuchâtel, Switzerland.

${ }^{16}$ Department of Ecology and Evolutionary Biology, University of California, 321 Steinhaus Hall, Irvine, California 92697-2525, USA.

${ }^{17}$ Centre for Ecology and Conservation, University of Exeter, Cornwall,

Tremough, Penryn TR10 9EZ, UK.

${ }^{18}$ Department of Ecology and Evolution, Biophore, University of Lausanne, 1015 Lausanne, Switzerland.

${ }^{19}$ Department of Biology, 167 Castetter Hall, MSC03 2020, 1 University of New Mexico, Albuquerque, New Mexico 87131-000, USA.

${ }^{20}$ Department of Zoology, University of Cambridge, Downing Street, Cambridge CB2 3EJ, UK.

${ }^{21}$ Evolution, Ecology and Genetics, Research School of Biology, Australian National University, Canberra, ACT 0200, Australia.

${ }^{22}$ Department of Biology and Biochemistry, University of Houston, Houston, Texas 77204-5001, USA.

${ }^{23}$ Institutes of Evolution, Immunology and Infection Research, School of Biological Sciences, Ashworth Laboratories, University of Edinburgh, Edinburgh EH9 3JT, UK. 


\section{BRIEF COMMUNICATIONS ARISING}

${ }^{24}$ Department of Psychology, University of California, Santa Barbara, Santa Barbara, California 93106-9660, USA.

${ }^{25}$ Department of Ecology and Evolutionary Biology, Princeton University, Princeton, New Jersey 08540, USA.

${ }^{26}$ Department of Ecology and Evolution, The University of Chicago, 1101 E. 57 Street, Chicago, Illinois 60637, USA.

${ }^{27}$ Department of Ecology, Montana State University, Bozeman, Montana 59717, USA

${ }^{28}$ Department of Biosciences, 8888 University Drive, Simon Fraser University, Burnaby, British Columbia V5A1S6, Canada.

${ }^{29}$ Department of Biology, Villanova University, 800 Lancaster Avenue, Villanova, Pennsylvania 19085, USA.

${ }^{30}$ Department of Mathematics and Statistics, Queen's University, Kingston, Ontario K7L 3N6, Canada.

${ }^{31}$ The Cornell Laboratory of Ornithology, Cornell University - The Johnson Center, 159 Sapsucker Woods Road, Ithaca, New York 14850, USA.

${ }^{32}$ Department of Biology, University of Louisville, Louisville, Kentucky 40292, USA

${ }^{33}$ Seeley G. Mudd Hall, Department of Neurobiology and Behavior, Cornell University, Ithaca, New York 14853, USA.

${ }^{34}$ USDA-ARS Bee Research Laboratory, BARC-E BIdg 476, Beltsville, Maryland 20705, USA.

${ }^{35}$ Laboratoire Ecologie and Evolution, CNRS UMR 7625, Ecole Normale Superieure, 46 rue d'UIm, 75005 Paris, France; Department of Ecology and Evolutionary Biology, University of Arizona, Tucson, Arizona 85721, USA.

${ }^{36}$ School of Life Sciences, John Maynard Smith Building, University of Sussex, Brighton BN1 9QG, UK.

${ }^{37}$ Department Biologie II Behavioral Ecology (Verhaltensökologie),

Ludwig-Maximilians-Universität, München Großhaderner Str. 2

D - 82152 Planegg/Martinsried, Germany.

${ }^{38}$ Department of Entomology, University of Kentucky, Lexington, Kentucky 40546-0091, USA.

${ }^{39}$ School of Life Sciences, Arizona State University, PO Box 874501,

Tempe, Arizona 85287-4501, USA.

${ }^{40}$ CEFE - UMR 5175, 1919 route de Mende, F-34293 Montpellier Cedex 5 , France.

${ }^{41}$ School of Biological Sciences, Flinders University, GPO Box 2100,

Adelaide, South Australia 5001, Australia.

${ }^{42}$ Kellogg Biological Station and Department of Zoology, Michigan State University, Hickory Corners, Michigan 49060, USA.

${ }^{43}$ School of Biology and Petit Institute for Bioengineering and Bioscience, Georgia Institute of Technology, 310 Ferst Drive, Atlanta, Georgia 30332-0230, USA.

${ }^{44}$ Department of Evolution and Ecology, College of Biological Sciences, 1 Shields Avenue, UC Davis, Davis, California 95616, USA.

${ }^{45}$ Center for Pollinator Research, Huck Institutes of the Life Sciences, Pennsylvania State University, Chemical Ecology Lab 4A, University Park, Pennsylvania 16802, USA.

${ }^{46}$ Muséum National d'Histoire Naturelle, CP39, 12 rue Buffon, 75005 Paris, France.

${ }^{47}$ Biology Department, University of Toronto, 3359 Mississauga Road, Mississauga, Ontario L5L 1C6, Canada.

${ }^{48}$ Department of Animal and Plant Sciences, University of Sheffield,

Western Bank, Sheffield S10 2TN, UK.

${ }^{49}$ Biologie I, Universität Regensburg, D-93040 Regensburg, Germany.

${ }^{50}$ Department of Biosciences, PL 65 (Viikinkaari 1), FI-00014 University of Helsinki, Finland.

${ }^{51}$ Department of Biology, University of Vermont, Burlington, Vermont 05405, USA

${ }^{52}$ School of Human Evolution and Social Change, Arizona State University, Tempe, Arizona 85287-2402, USA.
${ }^{53}$ Department of Animal Ecology, Institute of Ecological Science, Faculty of Earth and Life Sciences, Vrije Universiteit, De Boelelaan 1085, NL-1081 HV Amsterdam, The Netherlands.

${ }^{54}$ Animal Ecology Group, Centre for Evolutionary and Ecological Studies, University of Groningen, PO Box 14, 9750 AA Haren, The Netherlands.

${ }^{55}$ University of Osnabrueck, Barbarastr.11, D-49076 Osnabrueck, Germany.

${ }^{56}$ Harvard University, Museum of Comparative Zoology, 26 Oxford St, Cambridge, Massachusetts 02138, USA.

${ }^{57}$ Environmental Microbiology, Swiss Federal Institute of Aquatic Research and Technology, Überlandstrasse 133, CH-8600 Dübendorf, Switzerland.

${ }^{58}$ Centre for Social Evolution, Department of Biology, University of Copenhagen, Universitetsparken 15, DK-2100 Copenhagen, Denmark.

${ }^{59}$ School of Biological Sciences, Royal Holloway, University of London, Egham TW20 OEX, UK.

${ }^{60}$ Department of Ecology and Evolutionary Biology, University of

California, Santa Cruz, California 95064, USA.

${ }^{61}$ Department of Computer Science, University of Sheffield, Sheffield S1 4DP, UK.

${ }^{62}$ Department of Anthropology and Center for Population Biology, UC Davis, Davis, California 95616, USA.

${ }^{63}$ Department of Ecology and Evolutionary Biology, University of Arizona, Tucson, Arizona 85721, USA.

${ }^{64}$ Department of Zoology, 730 Van Vleet Oval, University of Oklahoma, Norman, Oklahoma 73019, USA.

${ }^{65}$ Department of Biology, Queen's University, Kingston, Ontario K7L 3N6, Canada.

${ }^{66}$ Integrative Biology, University of Texas at Austin, 1 University Station C0930, Austin, Texas 78712, USA.

${ }^{67}$ Psychologie - Université de Strasbourg, Ethologie des Primates DEPE (IPHC CNRS UMR 7178), 23 rue Becquerel — Strasbourg 67087 , Cedex, France.

${ }^{68}$ Department of Philosophy, University of Bristol, Bristol BS8 1TB, UK.

${ }^{69}$ Biocenter Oulu and Department of Biosciences, University of Helsinki, Box 65, 00140 University of Helsinki, Finland

${ }^{70}$ Institute of Integrative Biology, Biosciences Building, Crown Street, University of Liverpool, Liverpool L69 7ZB, UK.

${ }^{71}$ Theoretical Biology group, University of Groningen, PO Box 14, 9750 AA Haren, The Netherlands.

${ }^{72}$ Department of Biology, CB\#3280, Coker Hall, University of North Carolina, Chapel Hill, NC 27599-3280, USA

${ }^{73}$ Department of Ecology and Evolutionary Biology, Rice University, Houston, Texas 77005-1892, USA

${ }^{74}$ Department of Biochemistry, University of Zurich, Building Y27, Office J-46, Winterthurstrasse 190, CH-8057 Zurich, Switzerland; Swiss

Institute of Bioinformatics, Quartier Sorge Bâtiment Génopode, CH- 1015 Lausanne, Switzerland.

${ }^{75}$ Research Department of Genetics, Evolution and Environment, Faculty of Life Sciences, University College London, 4 Stephenson Way, London NW1 2HE, UK.

${ }^{76}$ Centre for Behaviour and Evolution, Institute of Neuroscience, Faculty of Medical Sciences, Newcastle University, Henry Wellcome Building, Framlington Place, Newcastle upon Tyne NE2 4HH, UK.

${ }^{77}$ School of Marine and Tropical Biology, James Cook University, Queensland 4811, Australia.

${ }^{78}$ Station Biologique de Roscoff, CNRS-UPMC UMR 7144, 29680

Roscoff, France.

${ }^{79}$ Institut des Sciences de l'Evolution, University of Montpellier 2, Montpellier 34095, France.

${ }^{80}$ Department of Biology, University of North Carolina at Greensboro, 312 Eberhart Building, Greensboro, North Carolina 27403, USA. 
${ }^{81}$ Department of Biology, 3314 Spieth Hall, University of California Riverside, Riverside, California 92521, USA.

${ }^{82}$ ETH Zurich, Institute of Integrative Biology (IBZ), Universitätsstrasse 16, CH.8092 Zürich, Switzerland.

${ }^{83}$ School of Philosophy, Psychology and Language Sciences, University of Edinburgh, 3 Charles Street, Edinburgh EH8 9AD, UK.

${ }^{84}$ School of Biology, University of St Andrews, Harold Mitchell Building, St Andrews, Fife KY16 9TH, UK.

${ }^{85}$ William Paterson University of New Jersey, 300 Pompton Road, Wayne, New Jersey 07470, USA.

${ }^{86}$ Department of Anthropology, 101 West Hall, University of Michigan, Ann Arbor, Michigan 48109, USA.

${ }^{87}$ Department of Entomology and Department of Animal Biology,

University of Illinois, Urbana, Illinois 61801, USA.

${ }^{88}$ Behavioural Ecology, Institute of Ecology and Evolution, University of Bern, Wohlenstrasse 50a, CH-3032 Hinterkappelen, Switzerland.

${ }^{89}$ Department of Biology, University of Western Ontario, 1151 Richmond

Street North, London, Ontario N6A 5B7, Canada.

${ }^{90}$ Department of Anthropology, University of California, Santa Barbara, California 93106-3210, USA.

${ }^{91}$ Deptartment of Environmental Science, Policy and Management, 130 Mulford Hall, 3114, University of California Berkeley, Berkeley, California 94720-3114, USA.

${ }^{92}$ Faculty of Agriculture, University of the Ryukyus, Okinawa 903-0213, Japan.

${ }^{93}$ Dipartimento di Biologia Evoluzionistica, Università degli Studi di Firenze, via Romana 17, 50125 Firenze, Italy.

${ }^{94}$ Department of Ecology and Evolutionary Biology, University of

Tennessee Knoxville, Knoxville, Tennessee 37902, USA.

${ }^{95}$ Department of Entomology, Box 7613, North Carolina State University, Raleigh, North Carolina 27695-7613, USA.

${ }^{96}$ Institute for Theoretical Biology, Humboldt University zu Berlin, Invalidenstr. 43, D-10115 Germany.

${ }^{97}$ Departmet of Biology, Zoological Institute, K.U. Leuven, Naamsestraat 59, B-3000 Leuven, Belgium.

${ }^{98}$ Smithsonian Tropical Research Institute, Apartado 0843-03092, Balboa, Panamá.

${ }^{99}$ Department of Biology, 101 Morgan Building, University of Kentucky, Lexington, Kentucky 40506-0225, USA.
${ }^{100}$ Department of Applied Mathematics, University of Western Ontario, 1151 Richmond Street North, London, Ontario N6A 5B7, Canada.

${ }^{101}$ Department of Human Evolutionary Biology, Harvard University, Cambridge, Massachusetts 02138, USA.

${ }^{102}$ Department of Biology and Program in Ecology, Evolution and Conservation Biology, University of Nevada, Reno, Nevada 89557, USA.

${ }^{103}$ Department of Biology, San Francisco State University, San Francisco, California 94132, USA.

\section{Received 20 September; accepted 17 December 2010.}

1. Nowak, M. A., Tarnita, C. E. \& Wilson, E. O. The evolution of eusociality. Nature 466, 1057-1062 (2010).

2. Hamilton, W. D. The genetical evolution of social behaviour, I \& II. J. Theor. Biol. 7, 1-52 (1964).

3. Hamilton, W. D. Selfish and spiteful behaviour in an evolutionary model. Nature 228, 1218-1220 (1970).

4. Grafen, A. Optimisation of inclusive fitness. J. Theor. Biol. 238, 541-563 (2006).

5. Frank, S. A. Foundations of Social Evolution (Princeton Univ. Press, 1998).

6. Gardner, A., West, S. A. \& Barton, N. H. The relation between multilocus population genetics and social evolution theory. Am. Nat. 169, 207-226 (2007).

7. Rousset, F. Genetic Structure and Selection in Subdivided Populations (Princeton Univ. Press, 2004).

8. Queller, D. C. A general model for kin selection. Evolution 46, 376-380 (1992).

9. Krebs, J. R. \& Davies, N. B. Behavioural Ecology. An Evolutionary Approach 4th edn (Blackwell Scientific, 1997).

10. Foster, K. R. A defense of sociobiology. Cold Spring Harb. Symp. Quant. Biol. 74, 403-418 (2009).

11. Westneat, D. F. \& Fox, C. W. Evolutionary Behavioral Ecology (Oxford Univ. Press, 2010).

12. Hardy, I. C. W. Sex Ratios: Concepts and Research Methods (Cambridge Univ. Press, 2002).

13. West, S. A. Sex Allocation (Princeton Univ. Press, 2009).

14. Queller, D. C. \& Strassmann, J. E. Kin selection and social insects. Bioscience 48, 165-175 (1998).

15. Boomsma, J. J. Lifetime monogamy and the evolution of eusociality. Phil. Trans. $R$. Soc. Lond. B 364, 3191-3207 (2009).

16. Ratnieks, F. L. W., Foster, K. R. \& Wenseleers, T. Conflict resolution in insect societies. Annu. Rev. Entomol. 51, 581-608 (2006).

Author Contributions All authors contributed to the planning, writing and/or revising of this paper. Several others who contributed significantly are not listed because they are named on separate comments.

Competing financial interests: declared none.

doi:10.1038/nature09831

\section{Only full-sibling families evolved eusociality}

ARISING FRom M. A. Nowak, C. E. Tarnita \& E. O. Wilson Nature 466, 1057-1062 (2010)

The paper by Nowak et al. ${ }^{1}$ has the evolution of eusociality as its title, but it is mostly about something else. It argues against inclusive fitness theory and offers an alternative modelling approach that is claimed to be more fundamental and general, but which, we believe, has no practical biological meaning for the evolution of eusociality. Nowak et al. ${ }^{1}$ overlook the robust empirical observation that eusociality has only arisen in clades where mothers are associated with their full-sibling offspring; that is, in families where the average relatedness of offspring to siblings is as high as to their own offspring, independent of population structure or ploidy. We believe that this omission makes the paper largely irrelevant for understanding the evolution of eusociality.

Eusociality is not just any form of condition-dependent reproductive altruism as found in cooperative breeders, but the permanent division of reproductive labour. Clades where helpers became irreversibly eusocial (ants, some bees, some wasps, and termites ${ }^{2}$ ) are old, radiated into many subclades over evolutionary time, and achieved considerable ecological footprints. A recent comparative study ${ }^{3}$ showed that all hymenopteran clades that fit the standard definition of eusociality ${ }^{4}$ evolved from lifetime monogamous ancestors ${ }^{5-8}$. This implies that high relatedness always preceded or coincided with eusociality, and contrasts with the contention of Nowak et al. ${ }^{1}$ that eusociality can evolve in any group with parental care, or that high relatedness arises after eusociality.

Given that promiscuity is the most common mating system in animals, strict ancestral monogamy throughout eusocial clades implies that high relatedness was necessary for eusociality to evolve. Nonetheless, necessity does not imply sufficiency. Monogamous lineages may have remained solitary because the benefits of helping at the nest were insufficient to surpass independent breeding. This is elegantly captured by the ratio of the parameters $b$ and $c$ in Hamilton's rule. In a number of ant, bee and wasp genera the high relatedness condition for eusociality has become secondarily relaxed via evolutionary elaborations such as multiple queen mating, but this has only occurred after worker phenotypes had specialized so that opting out to independent breeding had become selectively disadvantageous or 
developmentally impossible ${ }^{3}$. Claiming (in their Supplementary Information, Part B) that it is far simpler to consider that advanced eusocial species just need more sperm ${ }^{1}$ muddles proximate and ultimate explanations ${ }^{9,10}$; many multiply-mating queens discard most of the sperm they receive $\mathrm{e}^{11,12}$, indicating that sperm limitation cannot explain polyandry.

We now also know that departures from high relatedness would almost certainly have prevented the evolution of eusociality if they had happened before sterile castes had become permanent ${ }^{8}$, that is, before reaching the point of no return to breeding independently ${ }^{13}$. A recent comparative study on birds ${ }^{14}$ showed that cooperative breeding is an unstable state that predominantly occurs in monogamous clades and is likely to be lost when parents become more promiscuous. This evidence is not merely correlative: differences in ancestral promiscuity between cooperative and non-cooperative species were found even before cooperation arose, illustrating that monogamy preceded the evolution of helping and that helpers leave when relatedness incentives are reduced. This shows that high relatedness among siblings is critical along with the Hamiltonian $b / c$ ratio but, as in the insects, relatedness is not sufficient because many monogamous birds are not cooperative breeders.

In light of these reconstructions of the ancestral life histories of numerous social clades, it is surprising that the argument of Nowak et al. ${ }^{1}$ about eusocial evolution starts by assuming that family structure can be replaced by any form of population structure. This assumption is puzzling given the lack of empirical evidence that this hypothetical 'parasocial' route to eusociality ${ }^{1,4}$ (where same-generation individuals associate independent of relatedness) has produced a single extant clade with obligately eusocial workers. We believe that this renders Part A of the Supplementary Information of Nowak et al. ${ }^{1}$, and the arguments throughout the first two-thirds of the paper, largely irrelevant to the origin of eusociality. Part C of the Supplementary Information addresses the evolution of sterile workers within monogamous or clonal families, meaning that relatedness in these models is invariant. As a consequence, we believe that these models have nothing to say about the importance of relatedness in the evolution of eusociality beyond showing that costs and benefits are also important. This was already clear from Hamilton's rule nearly half a century ago.

It should give pause for thought that none of the long-recognized approximations of inclusive fitness theory raised in the paper was important enough to preclude kin selection theory from developing into a well-integrated network of complementary hypotheses with high predictive power for reproductive decision-making in real-world social organisms. In contrast, the abstractions of Nowak et al. ${ }^{1}$ fail to provide any new predictions or questions; all they apparently have to offer is the truism that helpers are associated with longer-lived, fecund breeders.

\author{
Jacobus J. Boomsma ${ }^{1}$, Madeleine Beekman ${ }^{2}$, Charlie K. Cornwallis ${ }^{3}$, \\ Ashleigh S. Griffin ${ }^{3}$, Luke Holman ${ }^{1}$, William O. H. Hughes ${ }^{4}$, \\ Laurent Keller ${ }^{5}$, Benjamin P. Oldroyd ${ }^{2}$ \& Francis L. W. Ratnieks ${ }^{6}$ \\ ${ }^{1}$ Centre for Social Evolution, Department of Biology, University of \\ Copenhagen, 2100 Copenhagen, Denmark. \\ e-mail: JJBoomsma@bio.ku.dk \\ ${ }^{2}$ Behaviour and Genetics of Social Insects Lab, School of Biological \\ Sciences A12, University of Sydney, New South Wales, Australia. \\ ${ }^{3}$ Department of Zoology, University of Oxford, South Parks Road, Oxford \\ OX1 3PS, UK. \\ ${ }^{4}$ Institute of Integrative and Comparative Biology, Miall Building, \\ University of Leeds, Leeds LS2 9JT, UK. \\ ${ }^{5}$ Department of Ecology and Evolution, Biophore, University of Lausanne, \\ 1015 Lausanne, Switzerland. \\ ${ }^{6}$ Laboratory of Apiculture and Social Insects, School of Life Sciences, \\ University of Sussex, Falmer, Brighton BN1 9QG, UK.
}

Received 19 September; accepted 17 December 2010.

1. Nowak, M. A., Tarnita, C. E. \& Wilson, E. O. The evolution of eusociality. Nature 466, 1057-1062 (2010)

2. Inward, D. J. G., Vogler, A. P. \& Eggleton, P. A comprehensive phylogenetic analysis of termites (Isoptera) illuminates key aspects of their evolutionary biology. Mol. Phylogenet. Evol. 44, 953-967 (2007).

3. Hughes, W. O. H., Oldroyd, B. P., Beekman, M. \& Ratnieks, F. L. W. Ancestral monogamy shows kin selection is key to the evolution of eusociality. Science $\mathbf{3 2 0}$ 1213-1216 (2008).

4. Wilson, E. O. The Insect Societies (Belknap Press of Harvard Univ. Press, 1971).

5. Hamilton, W. D. The genetical evolution of social behaviour, I \& II. J. Theor. Biol. 7, $1-52$ (1964).

6. Alexander, R. D. The evolution of social behavior. Annu. Rev. Ecol. Syst. 5, 325-383 (1974).

7. Charnov, E. L. Evolution of eusocial behavior: offspring choice or parental parasitism? J. Theor. Biol. 75, 451-465 (1978).

8. Boomsma, J. J. Kin selection versus sexual selection: Why the ends do not meet. Curr. Biol. 17, R673-R683 (2007).

9. Mayr, E. Cause and effect in biology. Science 134, 1501-1506 (1961).

10. Tinbergen, N. On aims and methods of ethology. Z. Tierpsychol. 20, 410-433 (1963).

11. Baer, B. Sexual selection in Apis bees. Apidologie (Celle) 36, 187-200 (2005).

12. den Boer, S. P. A. et al. Prudent sperm use by leaf-cutter ant queens. Proc. R. Soc. Lond. B 276, 3945-3953 (2009).

13. Wilson, E. O. One giant leap: How insects achieved altruism and colonial life. Bioscience 58, 17-25 (2008).

14. Cornwallis, C. K., West, S. A., Davis, K. E. \& Griffin, A. S. Promiscuity and the evolutionary transition to complex societies. Nature 466, 969-972 (2010).

Author Contributions J.J.B. took the initiative for this contribution and wrote the first draft. All co-authors provided written and/or oral comments that helped shape the final submission.

Competing financial interests: declared none.

doi:10.1038/nature09832

\title{
Kin selection and eusociality
}

\author{
ARISING from M. A. Nowak, C. E. Tarnita \& E. O. Wilson Nature 466, 1057-1062 (2010)
}

Hamilton ${ }^{1}$ described a selective process in which individuals affect kin (kin selection), developed a novel modelling strategy for it (inclusive fitness), and derived a rule to describe it (Hamilton's rule). Nowak et $a .^{2}$ assert that inclusive fitness is not the best modelling strategy, and also that its production has been "meagre". The former may be debated by theoreticians, but the latter is simply incorrect. There is abundant evidence to demonstrate that inclusive fitness, kin selection and Hamilton's rule have been extraordinarily productive for understanding the evolution of sociality.
Below we list a few examples of what has been learned from applying kin selection theory-there are thousands of others. (1) Organisms overwhelmingly direct costly assistance, and all true altruism, towards $\mathrm{kin}^{3}$. (2) Eusociality in insects originated in organisms with parental care and single mating, which means that relatedness among helpers and brood is generally at the level of siblings ${ }^{4}$. (3) Benefits that can make helping more profitable than reproducing independently often take the forms of either fortress defence (termites, naked mole rats, social shrimp, social thrips and aphids, and some ants) or life insurance 
(wasps, bees, other ants) ${ }^{5,6}$. (4) Sex ratios, worker egg laying, worker policing, caste conflict and other social interactions in eusocial insects are explained by kin selection theory ${ }^{5,7}$.

Kin selection evidence is not merely correlative. Numerous kin selection experiments manipulate relatedness in animals, plants and microbes. Other experiments manipulate costs and benefits and show that kin selection is predictive ${ }^{8}$.

Nowak et $a .^{2}$ say that the haplodiploid hypothesis is not the only explanation for eusociality, but that has not been in dispute for some time. Haplodiploidy is not necessary for the evolution of eusociality, and is not the same as kin selection ${ }^{5,7}$.

Kin selection does not explain all social behaviour, but the claim that it does has never been widely accepted. There are cooperative acts that benefit the actor directly, and between-species mutualisms that must have direct benefits to evolve ${ }^{9}$. But only kin selection can explain true altruism ${ }^{10}$.

Kin selection theory is still inspiring new research. Application of kin selection theory to microbes, including those causing human diseases, is expanding $^{11}$. Kin selection is changing our views of imprinting ${ }^{12}$ and maternal-fetus diseases in humans ${ }^{12}$.

Clearly kin selection is a strong, vibrant theory that is the basis for understanding how social behaviour has evolved. Perhaps the best examples come from kin recognition, a field that did not exist before Hamilton's insights ${ }^{7}$. We are puzzled why Nowak et al. ${ }^{2}$ would attack a body of research that has been exemplary as "a domain of empirical knowledge [that has] followed so closely and fruitfully upon an abstract theoretical idea."13.

\author{
Joan E. Strassmann ${ }^{1}$, Robert E. Page $\mathrm{Jr}^{2}$, Gene E. Robinson ${ }^{3}$ \& \\ Thomas D. Seeley ${ }^{4}$ \\ ${ }^{1}$ Department of Ecology and Evolutionary Biology, Rice University, \\ Houston, Texas, USA. \\ e-mail: strassm@rice.edu
}

${ }^{2}$ School of Life Sciences, Arizona State University, Tempe, Arizona, USA. ${ }^{3}$ Department of Entomology, and Institute for Genomic Biology, University of Illinois at Urbana-Champaign, Urbana, Illinois, USA. ${ }^{4}$ Department of Neurobiology and Behavior, Cornell University, Ithaca, New York, USA.

\section{Received 19 November; accepted 17 December 2010.}

1. Hamilton, W. D. The genetical evolution of social behaviour. I-II. J. Theor. Biol. 7, 1-52 (1964).

2. Nowak, M. A., Tarnita, C. E. \& Wilson, E. O. The evolution of eusociality. Nature 466, 1057-1062 (2010).

3. Griffin, A. S. \& West, S. A. Kin discrimination and the benefit of helping in cooperatively breeding vertebrates. Science 302, 634-636 (2003).

4. Hughes, W., Oldroyd, B., Beekman, M. \& Ratnieks, F. Ancestral monogamy shows kin selection is key to the evolution of eusociality. Science 320, 1213-1216 (2008).

5. Queller, D. C. \& Strassmann, J. E. Kin selection and social insects. Bioscience 48, $165-175$ (1998).

6. Strassmann, J. \& Queller, D. Insect societies as divided organisms: the complexities of purpose and cross-purpose. Proc. Natl Acad. Sci. USA 104, 8619-8626 (2007).

7. Crozier, R. H. \& Pamilo, P. Evolution of Social Insect Colonies: Sex Allocation and Kin Selection (Oxford Univ. Press, 1996).

8. Field, J. \& Cant, M. A. Social stability and helping in small animal societies. Phil. Trans. R. Soc. B 364, 3181-3189 (2009).

9. Sachs, J. L., Mueller, U. G., Wilcox, T. P. \& Bull, J. J. The evolution of cooperation. Q. Rev. Biol. 79, 135-160 (2004).

10. Lehmann, L. \& Keller, L. The evolution of cooperation and altruism. A general framework and a classification of models. J. Evol. Biol. 19, 1365-1376 (2006).

11. West, S. A., Diggle, S. P., Buckling, A., Gardner, A. \& Griffins, A. S. The social lives of microbes. Annu. Rev. Ecol. Evol. Syst. 38, 53-77 (2007).

12. Haig, D. Evolution of placental hormones. Am. J. Hum. Biol. 20, 222-223 (2008).

13. Wilson, E. O. in Kin Recognition in Animals (eds Fletcher, D. J. C. \& Michener, C. D.) 7-18 (Wiley, 1987).

Author Contributions All authors conceived the project, analysed the material and wrote the paper.

Competing financial interests: declared none.

doi:10.1038/nature09833

\title{
Inclusive fitness in evolution
}

\author{
ARISING From M. A. Nowak, C. E. Tarnita \& E. O. Wilson Nature 466, 1057-1062 (2010)
}

For over fifty years, the evolution of social behaviour has been guided by the concept of inclusive fitness as a measure of evolutionary success. Nowak et al. ${ }^{1}$ argue that inclusive fitness should be abandoned. In so doing, however, they misrepresent the role that inclusive fitness has played in the theory of social evolution by which understanding social behaviour in a variety of disciplines has developed and flourished. By discarding inclusive fitness on the basis of its limitations, they create a conceptual tension which, we argue, is unnecessary, and potentially dangerous for evolutionary biology.

The core argument of Nowak et $a l^{1}$. for abandoning inclusive fitness is its limited capacity to predict dynamics in evolutionary models. This is an old point, and one that was hotly debated in the early years of kin selection theory ${ }^{2}$. Inclusive fitness was developed by Hamilton to summarize a difficult frequency-dependent selection problem by using a simple maximization principle. Early work ${ }^{3}$ proved that the average inclusive fitness effect is maximized by behavioural evolution in family structured populations and that it provides the surface for the Wright's adaptive topography (arguably one of the most useful tools that has ever been developed for understanding evolution).

Hamilton's great insight was that individual fitness is not maximized by social evolution when relatives are present, inclusive fitness is. The idea that something other than the individual organism could be the fitness-maximizing unit was completely revolutionary at the time and opened new research areas that are still being developed, such as the study of transitions in units of evolution and individuality ${ }^{4}$.

Today, inclusive fitness and evolutionary dynamics models are bridged and linked by the unifying concept of invasion fitness $s^{5-11}$ (Fig. 1). Invasion fitness captures feedbacks between the evolution of social traits and the ecological structure of the evolving population $^{7-11}$ (Fig. 2). Invasion fitness embraces average fitness and inclusive fitness maximization at evolutionary steady states ${ }^{5,7,9-11}$, and further reveals the difficulty of reducing evolution to a simple maximization process $^{5,12}$ (Fig. 1a).

Under Hamilton's rule, the condition for invasion of an altruistic allele involves a linear function of genetic relatedness. With ecoevolutionary feedbacks, Hamilton's rule becomes part of a more complex and dynamic framework ${ }^{7,10,11}$ in which relatedness between interacting individuals is a dynamic property of the population and an outcome of the models, rather than a pre-defined feature (which relatedness was in earlier models of inclusive fitness). Yet in this new framework the assumption of weak selection alone is often sufficient for Hamilton's rule to predict accurately endpoints of altruism 
evolution $^{11}$ (Fig. 1b). This framework has also begun to provide the testable predictions under competing hypotheses about relatedness and sociality that Nowak et al. ${ }^{1}$ call for ${ }^{8-11}$ (Fig. 2).

Both inclusive fitness and average fitness maximization are general insights about the evolutionary process with great heuristic value, even though they rely on special conditions to predict evolutionary dynamics. Considerable progress in extending evolutionary dynamics models to more general ecological, behavioural and genetic scenarios has been guided by the inclusive fitness concept $t^{4,6-11,13-15}$. By opposing 'standard selection theory' and 'inclusive fitness theory', we believe that Nowak et al. ${ }^{1}$ give the incorrect (and potentially dangerous) impression that evolutionary thinking has branched out into conflicting and apparently incompatible directions. In fact, there is only one paradigm: natural selection driven by interactions, interactions of all kinds and at all levels. Inclusive fitness has been a powerful force in the development of this paradigm and is likely to have a continued role in the evolutionary theory of behaviour interactions.

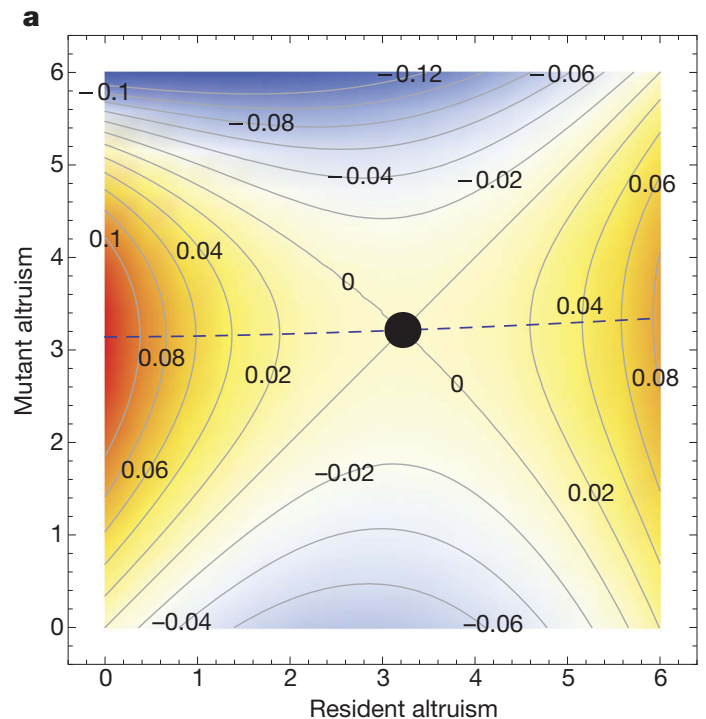

b

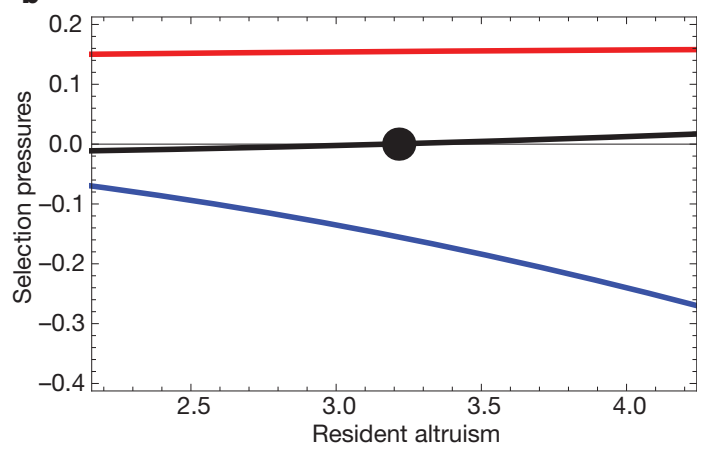

Figure $1 \mid$ Fitness landscape and Hamilton's rule. a, Fitness landscape in resident-mutant phenotypic space. Mutant invasion (inclusive) fitness is maximized along the dashed curve and the black circle is an evolutionarily stable phenotype. However, the asymmetrical sign structure implies that evolutionary dynamics do not obey an optimizing principle ${ }^{12}$. b. Selection pressures arise from relatedness (red curve), physiological cost of altruism (blue) and change in space occupancy (black). Under weak selection, the latter is negligible (black curve close to zero) and the benefit is proportional to relatedness (see for example, equation (3) in ref. 10), which makes Hamilton's rule a good approximation of selection ${ }^{10,11}$. The black circle indicates the evolutionarily stable phenotype found in a.

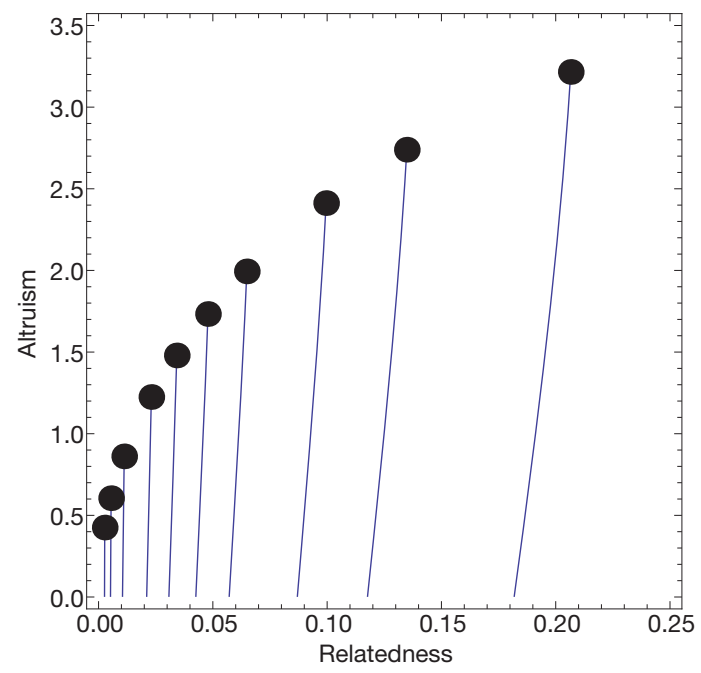

Figure $2 \mid$ Co-evolution of altruism and relatedness. Evolutionary trajectories (thin curves) start with zero altruism and converge to evolutionarily stable phenotypes (black circles). Relatedness is dynamic and co-evolves with altruism. Eco-evolutionary models can predict how environmental factors (for example, habitat viscosity) affect altruism evolution. Here, viscosity decreases across trajectories from right to left, and the string of black circles shows how evolved altruism and relatedness should co-vary along an environmental gradient of habitat viscosity.

\section{METHODS}

See ref. 10 for details on the model underlying Figs 1 and 2 (and others ${ }^{7,8,11}$ for further development). Altruism is a continuous character (with haploid inheritance) evolving in a spatial population network. Invasion fitness is the growth rate of a self-structured mutant cluster. Figure $1 \mathrm{a}, \mathrm{b}$ is based, respectively, on figures $6 \mathrm{E}$ and $4 \mathrm{E}$ in ref. 10 with mobility rate $=1$ and habitat viscosity $=1 / 4$. Figure 1a makes no special assumption on selection, interactions or population structure. Figure 2 is based on figures $5 \mathrm{~F}$ and $7 \mathrm{~F}$ in ref. 10 with mobility rate $=1$ and habitat viscosity from 1/4 (right) to 1/256 (left). Figures $1 \mathrm{~b}$ and 2 only assume weak selection.

\section{Regis Ferriere ${ }^{1,2}$ \& Richard E. Michod ${ }^{2}$ \\ ${ }^{1}$ Laboratoire Ecologie \& Evolution, CNRS UMR 7625, Ecole Normale \\ Superieure, 46 rue d'Ulm, 75005 Paris, France. \\ e-mail: regis.ferriere@ens.fr \\ ${ }^{2}$ Department of Ecology and Evolutionary Biology, University of Arizona, Tucson, Arizona 85721, USA.}

Received 27 September; accepted 17 December 2010.

1. Nowak, M. A., Tarnita, C. E. \& Wilson, E. O. The evolution of eusociality. Nature 466, 1057-1062 (2010).

2. Michod, R. E. The theory of kin selection. Annu. Rev. Ecol. Syst. 13, 23-55 (1982).

3. Michod, R. E. \& Abugov, R. Adaptive topography in family structured models of kin selection. Science 210, 667-669 (1980).

4. Michod, R. E. Darwinian Dynamics (Princeton Univ. Press, 1999).

5. Metz, J. A. J. in Encyclopedia of Ecology (eds Jorgensen, S. E. \& Fath, B. D.) (Elsevier, 2008).

6. Roff, D. A. Modeling Evolution (Oxford Univ. Press, 2010).

7. van Baalen, M. \& Rand, D. A. The unit of selection in viscous populations and the evolution of altruism. J. Theor. Biol. 193, 631-648 (1998).

8. Ferriere, R. \& Le Galliard, J.-F. in Dispersal (eds Clobert, J., Danchin, E., Dhondt, A. A. \& Nichols, J. D.) 57-79 (Cambridge Univ. Press, 2001).

9. Cadet, C., Ferriere, R., Metz, J. A. J. \& van Baalen, M. The evolution of dispersal under demographic stochasticity. Am. Nat. 162, 427-441 (2003).

10. Le Galliard, J.-F., Ferriere, R. \& Dieckmann, U. The adaptive dynamics of altruism in spatially heterogeneous populations. Evolution 57, 1-17 (2003).

11. Lion, S. \& van Baalen, M. From infanticide to parental care: why spatial structure can help adults be good parents. Am. Nat. 170, E26-E46 (2007).

12. Metz, J. A. J., Mylius, S. D. \& Diekmann, O. When does evolution optimize? Evol. Ecol. Res. 10, 629-654 (2008).

13. Michod, R. E. \& Sanderson, M. in Evolution-Essays in Honour of John Maynard Smith Ch. 7 (eds Greenwood, P. J. \& Slatkin, M.) 95-104 (Cambridge Univ. Press, 1985). 
14. Day, T.\& Taylor, P. D. Unifying genetics and game theoretic models of kin selection for continuous traits. J. Theor. Biol. 194, 391-407 (1998).

15. Rousset, F. Separation of time scales, fixation probabilities and convergence to evolutionarily stable states under isolation by distance. Theor. Popul. Biol. 69, 165-179 (2006).
Author Contributions R.F. and R.E.M. conceived the study. R.F. analysed the model and prepared the figures. R.F. and R.E.M. wrote the paper.

Competing financial interests: declared none.

doi:10.1038/nature09834

\title{
In defence of inclusive fitness theory
}

\author{
ARISING from M. A. Nowak, C. E. Tarnita \& E. O. Wilson Nature 466, 1057-1062 (2010)
}

Arguably the defining characteristic of the scientific process is its capacity for self-criticism and correction ${ }^{1}$. Nowak et al. ${ }^{2}$ challenge proposed connections between relatedness and the evolution of eusociality ${ }^{3}$, suggest instead that defensible nests and "spring-loaded" traits are key, and present alternative modelling approaches. They then dismiss the utility of Hamilton's insight that relatedness has a profound evolutionary effect ${ }^{3}$, formalized in his widely accepted inclusive fitness theory as Hamilton's rule ("Rise and fall of inclusive fitness theory"). However, we believe that Nowak et al. ${ }^{2}$ fail to make their case for logical, theoretical and empirical reasons.

Logically, both in attacking inclusive fitness and in attempting to reinforce their own positions, Nowak et al. ${ }^{2}$ cherry-pick examples and fail to distinguish necessary from sufficient causes ${ }^{1}$. Yes, there are hundreds of haplodiploid species that are not eusocial ${ }^{2}$. Yet, there are also hundreds of nest-making (diploid) birds, mammals and reptiles that are not eusocial. Moreover, if the non-eusocial, haplodiploid species pose a problem for inclusive fitness, then the fact that hundreds of them also make nests (including many living in communal or subsocial groups) does not support the proposed alternative.

Theoretically, in promoting their modelling approach, Nowak et al. ${ }^{2}$ pose a false dichotomy between inclusive fitness theory and "standard natural selection theory". They assert, we believe incorrectly, that inclusive fitness theory suffers from numerous ills (for example, "stringent assumptions"), yet their own models require stringent assumptions, without the benefit of any generality. Indeed, although asserting that "relatedness does not drive the evolution of eusociality", the authors do not present the critical test of removing the effects of relatedness in their model (for example, by randomly assigning daughters to nests). Thus, Nowak et al. ${ }^{2}$ do not provide any basis for their core assertion, and available data on real biological systems ${ }^{4-6}$ directly contradict it.

Empirically, Nowak et $a l^{2}$, in our eyes, misinterpret relevant literature. Emphasizing progressive provisioning of food to immatures as a critical pre-adaptation (that is, a "spring-loaded" trait), they overlook taxa (for example, sweat bees) in which eusociality evolved repeatedly without progressive provisioning ${ }^{7,8}$. It has been suggested that eusociality might rapidly evolve ${ }^{9}$, but the statement by Nowak et al. ${ }^{2}$ that studies of forced sociality in Lasioglossum bees show that solitary bees will divide labour "in foraging, tunnelling, and guarding" is incorrect. Lasioglossum hemichalceum is social (communal), not solitary ${ }^{10}$, and the solitary Lasioglossum figueresi was studied in artificial arenas, not nests, so it was impossible for bees to forage, tunnel or guard ${ }^{11}$. Moreover, the small carpenter bees that Nowak et al. ${ }^{2}$ cite are in a genus (Ceratina) that contains no known obligately eusocial species, and only one species in which faculative eusociality occurs at high frequency ${ }^{12}$, indicating that even if "spring-loaded" traits exist, Nowak et al. ${ }^{2}$ have misidentified them.

What is clear is that neither haplodiploidy, nests, nor "springloaded" traits is sufficient for the evolution of eusociality. However, the most recent comparative evidence supports the basic prediction of inclusive fitness theory that, regardless of ploidy or the presence of nests or "spring-loaded" traits, high relatedness is key to the evolution of cooperative breeding and/or eusociality ${ }^{4-6}$. Any serious attempt to dismiss inclusive fitness theory must address the results of these important comparative studies ${ }^{4-6}$ directly.

Beyond its being completely integrated with "standard natural selection theory" "13, beyond extensive theoretical work showing that it is both flexible and robust ${ }^{13}$, beyond the fact that available evidence supports its fundamental prediction that high relatedness is key for the evolution of eusociality ${ }^{4-6}$, inclusive fitness theory has the virtue of making general, non-obvious predictions well beyond the issue of eusociality $^{4-6}$. Kin recognition and policing ${ }^{14,15}$, mother-fetus conflicts, and patterns of sex allocation (particularly in eusocial insects) stand out ${ }^{3,14,15}$. Collectively, those predictions have again and again been borne out in a vast comparative and experimental empirical literature (for example, refs $3-6,14,15$ ) that Nowak et al. ${ }^{2}$ nonetheless dismiss as "meagre" and "superficial". Nowak et al. ${ }^{2}$ present a provocative essay, but in their apparent rush to discard inclusive fitness theory, they present an alternative that we believe to be deeply flawed. Although the continued scrutiny of accepted paradigms is an essential part of the scientific process, the reports ${ }^{2}$ of the fall of inclusive fitness theory have been greatly exaggerated. If anything, Nowak et al. ${ }^{2}$ succeed in reminding us of the elegance and power of Hamilton's numerous insights and contributions ${ }^{3}$.

\section{Edward Allen Herre ${ }^{1}$ \& William T. Wcislo ${ }^{1}$}

${ }^{1}$ Smithsonian Tropical Research Institute, Apartado 0843-03092, Balboa, Republic of Panama; Smithsonian Tropical Research Institute, MRC 0580-06, unit 9100 Box 0948, DPO AA 34002-9998, USA. e-mail: herrea@si.edu

\section{Received 8 October; accept 17 December 2010.}

1. Mayr, E. The Growth of Biological Thought (Harvard Univ. Press, 1982).

2. Nowak, M. A., Tarnita, C. E. \& Wilson, E. O. The evolution of eusociality. Nature 466 , 1057-1062 (2010).

3. Hamilton, W. D. Narrow Roads of Gene Land. Vol. I. Evolution of Social Behaviour (Oxford Univ. Press, 1998).

4. Hughes, W. O. H., Oldroyd, B. P., Beekman, M. \& Ratnieks, F. L. W. Ancestral monogamy shows kin selection is key to the evolution of eusociality. Science $\mathbf{3 2 0}$, 1213-1216 (2008)

5. Cornwallis, C. K., West, S. A., Davis, K. E. \& Griffin, A. S. Promiscuity and the evolution to complex societies. Nature 466, 969-972 (2010).

6. Boomsma, J. J. Lifetime monogamy and the evolution of eusociality. Phil. Trans. R. Soc. B 364, 3191-3207 (2009).

7. Costa, J. T. The Other Insect Societies (Harvard Univ. Press, 2006).

8. Michener, C. D. The Social Behavior of the Bees (Harvard Univ. Press, 1974).

9. Michener, C. D. From solitary to eusocial: Need there be a series of intervening species? Fortschr. Zool. 31, 293-306 (1985).

10. Jeanson, R. Kukuk, P. F. \& Fewell, J. H. Emergence of division of labour in halictine bees: Contributions of social interactions and behavioural variance. Anim. Behav. 70, 1183-1193 (2005).

11. Wcislo, W. T. Social interactions and behavioral context in a largely solitary bee, Lasioglossum (Dialictus) figueresi (Hymenoptera, Halictidae). Insectes Soc. 44, 199-208 (1997). 


\section{BRIEF COMMUNICATIONS ARISING}

12. Cronin, A. L. A molecular phylogeny and social behaviour of Japenese Ceratina (Hymenoptera, Apidae, Xylocopinae). Insect Syst. Evol. 35, 137-146 (2004).

13. Gardner, A., West, S. A. \& Barton, N. H. The relation between multilocus population genetics and social evolution theory. Am. Nat. 169, 207-226 (2007)

14. Ratnieks, F. L. W. \& Vissher, P. K. Worker policing in honeybees. Nature 342, 796-797 (1989).
15. Mueller, U. G. Haplodiploidy and the evolution of facultative sex ratios in a primitively eusocial bee. Science 254, 442-444 (1991).

Author Contributions Both authors contributed extensively to all aspects of this work.

Competing financial interests: declared none.

doi: $10.1038 /$ nature09835

\section{Nowak et al. reply}

REPLYING TO: P. Abbot et al. Nature 471, doi:10.1038/nature09831 (2011); J. J. Boomsma et al. Nature 471, doi:10.1038/nature09832 (2011); J. E. Strassmann et al. Nature 471, doi:10.1038/nature09833 (2011); R. Ferriere \& R. E. Michod Nature 471, doi:10.1038/nature09834 (2011); E. A. Herre \& W. T. Wcislo Nature 471, doi:10.1038/nature09835 (2011)

Our paper challenges the dominant role of inclusive fitness theory in the study of social evolution ${ }^{1}$. We show that inclusive fitness theory is not a constructive theory that allows a useful mathematical analysis of evolutionary processes. For studying the evolution of cooperation or eusociality we must instead rely on evolutionary game theory or population genetics. The authors of the five comments ${ }^{2-6}$ offer the usual defence of inclusive fitness theory, but do not take into account our new results.

The definition of inclusive fitness given by Hamilton ${ }^{7}$ is as follows:

\begin{abstract}
"Inclusive fitness may be imagined as the personal fitness which an individual actually expresses in its production of adult offspring as it becomes after it has been first stripped and then augmented in a certain way. It is stripped of all components which can be considered as due to the individual's social environment, leaving the fitness which he would express if not exposed to any of the harms or benefits of that environment. This quantity is then augmented by certain fractions of the quantities of harm and benefit which the individual himself causes to the fitnesses of his neighbours. The fractions in question are simply the coefficients of relationship appropriate to the neighbours whom he affects: unity for clonal individuals, one-half for sibs, one-quarter for half-sibs, one-eighth for cousins,...and finally zero for all neighbours whose relationship can be considered negligibly small."
\end{abstract}

The concept of inclusive fitness assumes that the fitness of individuals can be split into additive components caused by individual actions. This approach rests on specific assumptions, which need not hold for any particular evolutionary process. Therefore inclusive fitness theory is not a general description of natural selection. In Part A of our Supplementary Information ${ }^{1}$ we provide a mathematical analysis to prove this point. If there are non-zero selection intensities, or if there are synergistic interactions, or if there is complex population structure, then it is easy to find situations where personal fitness cannot be partitioned into additive components as needed by inclusive fitness theory. Essentially, inclusive fitness theory requires fitness to be a linear function of individual actions, but a full understanding of social evolution must take into account the nonlinearity inherent in biological systems.

We distinguish between inclusive fitness theory and standard natural selection theory, because the latter does not require fitness to be split into additive components. We have shown that inclusive fitness theory is a proper subset of the standard theory and makes no independent predictions. Any effect of relatedness is fully captured by the standard approach.

Hamilton's rule states that cooperation can evolve if relatedness exceeds the cost to benefit ratio. If cost and benefit are parameters of individual actions then this rule almost never holds ${ }^{1,8,9}$. There are attempts to make Hamilton's rule work by choosing generalized cost and benefit parameters ${ }^{10}$, but these parameters are no longer properties of individual phenotypes. They depend on the entire system including population structure. These extended versions of Hamilton's rule have no explanatory power for theory or experiment ${ }^{11}$.

Neither inclusive fitness theory nor any formulation of Hamilton's rule can deal with evolutionary dynamics ${ }^{12}$. This fact alone invalidates the claim that inclusive fitness theory "is as general as the genetical theory of natural selection" 2 .

Several aspects of our paper are misrepresented in the comments ${ }^{2-6}$. One, we do not argue that relatedness is unimportant. Relatedness is an aspect of population structure, which affects evolution ${ }^{13}$. Two, we do not dispute the importance of kin recognition. Conditional behaviour based on kin recognition can be seen as a mechanism for the evolution of cooperation ${ }^{14}$. Three, Part A of our Supplementary Information ${ }^{1}$ is not a model for evolution of eusociality, but a mathematical framework that demonstrates the limitations of inclusive fitness theory. Four, Part C of our Supplementary Information ${ }^{1}$ provides a mathematical model for the evolution of eusociality, which makes simple and testable predictions and explains the rarity of the phenomenon. Five, monogamy and sex ratio manipulation may be important for the evolution of eusociality; such ideas are best tested in the context of the explicit model that we propose.

Abbot et al. ${ }^{2}$ claim that inclusive fitness theory has been tested in a large number of biological contexts, but in our opinion this is not the case. We do not know of a single study where an exact inclusive fitness calculation was performed for an animal population and where the results of this calculation were empirically evaluated. Fitting data to generalized versions of Hamilton's rule is not a test of inclusive fitness theory, which is not even needed to derive such rules.

The limitations of inclusive fitness theory are also demonstrated by its inability to provide useful calculations for microbial evolution ${ }^{15,16}$.

Herre and Wcislo ${ }^{6}$ have presented a one-sided account of cases in halictid eusociality, the details of which do not detract in the least from our argument. Halictid bees were not ignored as stated; we cited them three times. Furthermore, communal halictid bees are 'social' only in a primitive sense. They occupy a commons-like tunnel but build and defend their own personal cells as solitary bees ${ }^{17}$. Herre and Wcislo ${ }^{6}$ point out that the experiments of $\mathrm{Wcislo}^{18}$ were designed not to allow foraging, tunnelling, or guarding, but do not mention that these behaviours were tested in other experiments ${ }^{19,20}$. Bees are mass provisioners, as Herre and Wcislo ${ }^{6}$ say, and we should have used the phrase 'defence and care of young with mass provisioning (bees) or progressive provisioning (others)'. We thank Herre and Wcislo ${ }^{6}$ for pointing out this oversight. Primitively eusocial halictids nevertheless devote considerable care to the cells, guarding them and in many cases opening them to clean out waste.

Various authors mention sex ratio theory, which we do not study in our paper. Nevertheless a precise understanding of sex ratio evolution is based on population genetics and does not require inclusive fitness theory. 
There is no support for the claim that evolution maximizes inclusive fitness. Nobody has offered a mathematical statement explaining what should be maximized and for which process.

Hamilton's work has stimulated much empirical research and has led to many measurements of relatedness. But we have shown that we cannot rely on inclusive fitness theory to describe how interactions among related individuals affect evolution. Inclusive fitness theory is neither useful nor necessary to explain the evolution of eusociality or other phenomena. It is time for the field of social evolution to move beyond the limitations of inclusive fitness theory.

\section{Martin A. Nowak ${ }^{1}$, Corina E. Tarnita ${ }^{1}$ \& Edward O. Wilson ${ }^{2}$}

${ }^{1}$ Program for Evolutionary Dynamics, Department of Mathematics, Department of Organismic and Evolutionary Biology, Harvard University, Cambridge, Massachusetts 02138, USA.

e-mail: martin_nowak@harvard.edu

${ }^{2}$ Museum of Comparative Zoology, Harvard University, Cambridge,

Massachusetts 02138, USA.

1. Nowak, M. A., Tarnita, C. E. \& Wilson, E. O. The evolution of eusociality. Nature 466 , 1057-1062 (2010)

2. Abbot, P. et al. Inclusive fitness theory and eusociality. Nature 471, doi:10.1038/ nature09831 (2011)

3. Boomsma, J. J. et al. Only full-sibling families evolved eusociality. Nature 471, doi:10.1038/nature09832 (2011).

4. Strassmann, J. E., Page, R. E. Jr, Robinson, G. E. \& Seeley, T. D. Kin selection and eusociality. Nature 471, doi:10.1038/nature09833 (2011).

5. Ferriere, R. \& Michod, R. E. Inclusive fitness in evolution. Nature 471, doi:10.1038/ nature09834 (2011)
6. Herre, E. A. \& Wcislo, W. T. In defence of inclusive fitness theory. Nature $\mathbf{4 7 1}$, doi:10.1038/nature09835 (2011).

7. Hamilton, W. D. The genetical evolution of social behaviour, I, II. J. Theor. Biol. 7, 1-52 (1964)

8. Cavalli-Sforza, L. L. \& Feldman, M. W. Darwinian selection and "altruism". Theor. Popul. Biol. 14, 268-280 (1978).

9. Karlin, S. \& Matessi, C. Kin selection and altruism. Proc. R. Soc. Lond. B 219, 327-353 (1983).

10. Queller, D. C. A general model for kin selection. Evolution 46, 376-380 (1992).

11. Chuang, J. S., Rivoire, O. \& Leibler, S. Cooperation and Hamilton's rule in a simple synthetic microbial system. Mol. Syst. Biol. 6, 398 (2010).

12. Traulsen, A. Mathematics of kin- and group-selection: Formally equivalent? Evolution 64, 316-323 (2010)

13. Nowak, M. A., Tarnita, C. E. \& Antal, T. Evolutionary dynamics in structured populations. Phil. Trans. R. Soc. B 365, 19-30 (2010).

14. Nowak, M. A. Five rules for the evolution of cooperation. Science 314, 1560-1563 (2006).

15. Diggle, S. P., Griffin, A. S., Campell, G. S. \& West, S. A. Cooperation and conflict in quorum-sensing bacterial populations. Nature 450, 411-414 (2007).

16. Smith, J., van Dyken, J. D. \& Zee, P. C. A generalization of Hamilton's rule for the evolution of microbial cooperation. Science 328, 1700-1703 (2010).

17. Michener, C. D. The Social Behavior of the Bees (Harvard Univ. Press, 1974).

18. Wcislo, W. T. Social interactions and behavioral context in a largely solitary bee, Lasioglossum (Dialictus) figueresi (Hymenoptera, Halictidae). Insectes Soc. 44, 199-208 (1997).

19. Jeanson, R., Kukuk, P. F. \& Fewell, J. H. Emergence of division of labour in halictine bees: Contributions of social interactions and behavioural variance. Anim. Behav. 70, 1183-1193 (2005).

20. Sakagami, S. F. \& Maeta, Y. Sociality, induced and/or natural, in the basically solitary small carpenter bees (Ceratina). In Animal Societies: Theories and Facts (eds Itô, Y., Brown, J. L. \& Kikkawa, J.) 1-16 (Japan Scientific Societies Press, 1987).

Author Contributions M.A.N., C.E.T. and E.O.W. collaborated on all aspects of this reply. Competing financial interests: declared none.

doi: $10.1038 /$ nature09836 